(2) Open Access Full Text Article

\title{
Patterns of local failures and suggestions for reduction of clinical target volume for nasopharyngeal carcinoma patients without cervical lymph node metastasis
}

This article was published in the following Dove Press journal:

OncoTargets and Therapy

Yujiao $\mathrm{Li}^{1,2, *}$

Xiaomin Ou ${ }^{1,2, *}$

Chunying Shen ${ }^{1,2}$

Tingting $\mathrm{Xu}^{1,2}$

Weiwei $\mathrm{Li}^{1,2}$

Chaosu $\mathrm{Hu}^{1,2}$

'Department of Radiation Oncology, Fudan University Shanghai Cancer

Center, Shanghai, China; ${ }^{2}$ Department of Oncology, Shanghai Medical

College, Shanghai, China

*These authors contributed equally to the paper
Correspondence: Chaosu Hu Fudan University Shanghai Cancer

Center, 270 Dong An Road, Shanghai 200032, China

Tel +86 2I 64175590 ext 1400

Fax +86 2I 64I74774

Email hucsu62@yahoo.com
Background: To demonstrate the robustness of clinical target volume delineation for nasopharyngeal carcinoma (NPC) patients, this study makes a detailed analysis of the initial irradiated dose of the recurrent site and local failure patterns after intensity-modulated radiation therapy (IMRT). Based on this analysis, further improvement of delineation recommendations may be made in order to improve the quality-of-life in NPC, without decreasing the local control and survival rate.

Methods: In total, 382 newly diagnosed non-metastatic NPC patients were retrospectively enrolled, receiving elective neck irradiation to levels II, III, and VA. For patients with local failure, the location and extent of local failures were transferred to the pretreatment planning computed tomography (CT) for dosimetric analysis. The dose of radiation received by GTVr (gross tumor volume of recurrence) was calculated and analyzed with dose-volume histogram (DVH). Failures were classified as: "in field" if 95\% of GTVr was within the $95 \%$ isodose, "marginal" if $20 \%-95 \%$ of GTVr was within the $95 \%$ isodose, or "outside" if less than $20 \%$ of GTVr was inside the $95 \%$ isodose.

Results: With a median follow-up time of 61.3 months, 12 patients developed local recurrence ( 10 cases available). The 5-year overall survival, local relapse-free survival, regional relapse-free survival, distant metastasis failure-free survival, and disease-free survival were $87.8 \%, 95.2 \%, 99.1 \%, 93.3 \%$, and $82.5 \%$, respectively. Dose conformity with IMRT was excellent, and the recurrence was mainly within 3 years after the first treatment. The dosimetric analysis showed that seven failures were classified as "in-field", two failures as "marginal", and only one failure as "out-field". Most local relapse sites located just the same site of primary tumor and most anatomic sites were at low risk of concurrent bilateral tumor invasion. Conclusions: IMRT with elective neck irradiation provides excellent local control for NPC patients without cervical lymph node metastasis. In-field failures are the main patterns for local recurrence, and the radioresistant subvolumes within the gross tumor volume are needed to be identified. This study proposed suggestions for reduction of target volume during IMRT treatment for NPC patients.

Keywords: nasopharyngeal carcinoma, intensity-modulated radiotherapy, patterns of local failure, reduction of clinical target volume

\section{Introduction}

Nasopharyngeal carcinoma (NPC) is the most common head and neck malignancy in Southeast Asia, and it is highly sensitive to radiotherapy (RT) or chemotherapy. ${ }^{1}$ Intensity-modulated radiation therapy (IMRT) has been widely applied in the field of 
radiation oncology over the last decade, and is considered as a major breakthrough for NPC, due to its capability of delivering a high radiation dose to the target, while sparing the adjacent organs. ${ }^{2,3}$

The application of IMRT and systemic therapy has greatly improved the local control of NPC. However, local recurrence still accounted for $60 \%$ of failures among patients with locoregional advanced disease. ${ }^{4}$ The management of post-treatment recurrence remains an intractable issue. In 2000, Dawson et $\mathrm{al}^{5}$ first analyzed the relationship of the recurrent region to the previously treated dose distribution and, with a median follow-up of 27 months, they found that the majority of local recurrences after conformal and segmental IMRT were "in-field," in areas which were judged to be at high risk at the time of RT planning, including the gross tumour volume (GTV) and the operative bed. Over the past few years, many studies reported similar failure patterns in NPC patients., ${ }^{4,6-8}$ On the other hand, Lin et $\mathrm{al}^{9}$ reported similar local control in a series of NPC patients retreated with reduced-volume IMRT, compared with results from another institution with a larger clinical tumor volume. This suggested the potential of reducing clinical target volume (CTV), meanwhile not impairing local control.

Nevertheless, IMRT planning is usually associated with sharp dose gradients outside the target volumes; therefore, the importance of accurate target delineation at planning should be stressed. An inadequate definition of target volumes could increase the risk of geographic misses, which eventually leads to local recurrence. ${ }^{10} \mathrm{We}$ assume the analysis of local failure patterns is essential because it is concerned with the evaluation of the quality of target volume delineation.

In our study, we make a detailed analysis of the initial irradiated dose of the recurrent site and local failure patterns, with the aim to demonstrate the robustness of CTV delineation. Based on this analysis, further improvement of delineation recommendation may be made in order to improve the quality-of-life in NPC, without decreasing the local control and survival rate.

\section{Methods}

\section{Patient and pretreatment evaluations}

In this retrospective analysis, data from 1,732 consecutive and nonselected histologically proven NPC patients were collected at Fudan University Shanghai Cancer Center between January 2009 and December 2011, and 382 patients had no clinical evidence of neck node metastasis according to 2002 American Joint Committee on Cancer (AJCC) staging criteria. The pretreatment magnetic resonance images were retrospectively reviewed to confirm proper staging, and all patients were restaged based on 2010 AJCC staging criteria. Hence, a portion of the patients with retropharyngeal lymph node involvement was diagnosed as N1, based on new staging criteria.

Pretreatment evaluation consisted of a complete history and physical examination, indirect or fiber-optic endoscopic examination, complete blood counts, determination of serum electrolytes, chest computed tomography (CT) scan or X-ray, magnetic resonance imaging (MRI) scan of the head and neck, ultrasound of the liver and abdomen, and dental evaluation. Urinalysis, bone scan, and positron emission tomography-computed tomography (PET-CT) were performed when clinically indicated.

\section{Ethical approval and informed consent}

All procedures performed in studies involving human participants were in accordance with the ethical standards of Fudan University Shanghai Cancer Center Ethics committee and with the 1964 Helsinki declaration and its later amendments or comparable ethical standards. The experimental protocols were also approved by Fudan University Shanghai Cancer Center Ethics committee. Written informed consent was obtained from all individual participants included in the study.

\section{Intensity-modulated radiotherapy}

\section{RT: immobilization and simulation}

All patients were immobilized in the supine position with a thermoplastic mask, followed by conventional simulation and planning. Intravenous contrast-enhanced CT, using a slice thickness of $5 \mathrm{~mm}$, was performed for planning. Image fusion of the T1 sequences with gadolinium enhanced MRI was performed with the CT simulation images for target delineation. The CT data were imported to the treatment planning system for treatment design. ${ }^{7}$

\section{RT: target volume delineation}

The target volumes were defined in accordance with the International Commission on Radiation Units and Measurements Reports. The primary gross tumour volume (GTV_P) included all gross tumours, and was determined by imaging, clinical, and endoscopic findings. The enlarged retropharyngeal nodes were outlined, together with primary GTV on the IMRT plans.

All received elective neck irradiation to levels II, III, and VA and the other node levels were spared. One CTV was defined in our radiotherapy: CTV1. The CTV1 was defined as the high-risk region that included GTV_P plus a 5-10 $\mathrm{mm}$ margin to take into account subclinical extension. 
CTV1 should also include the entire nasopharynx, skull base, parapharyngeal space, retropharyngeal lymph nodal regions, inferior sphenoid sinus, pterygoid fossae, clivus, the posterior third of the nasal cavity and maxillary sinuses, and any high risk nodal regions, including the bilateral upper deep jugular nodes, according to the current protocol in our center. ${ }^{2}$

The planning target volume (PTV_C) encompassed the CTV with a 3-mm margin in all directions. However, when the CTV was near the brainstem and spinal cord, PTV_C was generated with a margin less than $1 \mathrm{~mm}$.

The organs at risk include the brainstem, spinal cord, parotid glands, optic pathways, chiasm, eyeballs, lens, mandible, temporal lobes, inner ears, larynx, thyroid, and oral mucosa. A 5-mm margin was added to the spinal cord and at least a 1-mm margin was added to the brainstem during optimization to form the planning organ-at-risk volume.

\section{RT: treatment planning and delivery}

All patients were treated with external-beam radiation therapy using 6-MV photons, 7-9 radiation fields. The treatment technique used was the simultaneous integrated boost technique. The prescribed dose was $66 \mathrm{~Gy}$ in 30 fractions to planning target volume of primary tumor (PTV-G) for T1-2 and 70.4 Gy in 32 fractions for T3-4. The dose delivered to PTV_C for subclinical disease and regional lymphatics was 60 Gy at high risk in 30-32 fractions. All patients were treated with one fraction per day for 5 days per week. At least $95 \%$ of PTV volume received the prescription dose. The volume fraction receiving a dose less than $95 \%$ of the prescription dose did not exceed $1 \%$. No patients received more than $110 \%$ of the prescription dose into or out of the PTV. The dose received by each organ at risk was limited to tolerance, according to the Radiation Therapy Oncology Group (RTOG) 0225 protocol. ${ }^{11}$ The dose distribution was also examined slice-by-slice on the CT images.

\section{Chemotherapy}

Chemotherapy, including neoadjuvant chemotherapy, concurrent chemotherapy, and adjuvant chemotherapy, was given to patients when clinically indicated. The most common regimen of neoadjuvant and adjuvant chemotherapy included two to three cycles of TP (docetaxel $75 \mathrm{mg} / \mathrm{m}^{2} /$ day, day 1 , cisplatin $25 \mathrm{mg} / \mathrm{m}^{2} /$ day, days $1-3$ ), TPF (docetaxel $75 \mathrm{mg} / \mathrm{m}^{2} /$ day, day 1 , cisplatin $25 \mathrm{mg} / \mathrm{m}^{2} /$ day, days $1-3$, and 5-fluorouracil $2.5 \mathrm{~g} / \mathrm{m}^{2} \mathrm{civ} 120 \mathrm{~h}$ ), or GP (gemcitabine $1 \mathrm{~g} / \mathrm{m}^{2} /$ day, day 1 , day 8 , cisplatin $25 \mathrm{mg} / \mathrm{m}^{2} /$ day, days $1-3$ ) regimen. Induction chemotherapy was given every 3 weeks. Four weeks after the completion of RT, the adjuvant chemotherapy was administered every 3 weeks. Concurrent chemotherapy consisted of $80 \mathrm{mg} / \mathrm{m}^{2}$ of cisplatin every 3 weeks for $2-3$ cycles. ${ }^{2}$

\section{Patient evaluation and follow-up}

All patients were evaluated weekly for treatment response and toxicities during radiation therapy. After IMRT, patients were clinically evaluated every 3 months in the first 2 years, every 6 months from the third year to the fifth year, and annually thereafter. Each follow-up included examination of the nasopharynx and palpation of neck nodes. MRI of the nasopharynx was performed every half year, while a chest CT scan and ultrasound of the abdomen was scheduled annually after the completion of IMRT. Additional tests were ordered when indicated to evaluate local or distant relapse. Radiotherapy-related toxicities were graded according to the Acute and the Late Radiation Morbidity Scoring Criteria of RTOG. Chemotherapy-related toxicities were graded by the National Cancer Institute Common Toxicity Criteria (NCI-CTC) version 3.0.

\section{Definition of failure site}

All local failures were documented clinically and with appropriate imaging (MRI and 18F-fluorodeoxyglucose positron emission tomography) and, when possible, confirmed pathologically. For patients with local failures, the location and extent of failures were transferred to the pretreatment planning CT for dosimetric analysis. The dose of radiation received by GTVr (gross tumor volume of recurrence) was calculated and analyzed with dose-volume histogram (DVH). Failures were classified as: "in field" if 95\% of GTVr was within the $95 \%$ isodose, "marginal" if $20 \%-95 \%$ of GTVr was within the $95 \%$ isodose, or "outside" if less than $20 \%$ of GTVr was inside the $95 \%$ isodose. ${ }^{7}$

\section{Statistical analysis}

The follow-up time was calculated from the day of the completion of IMRT. The Statistical Package for Social Sciences (SPSS version 22.0) software was used for statistical analysis. The Kaplan-Meier method was used to evaluate the overall survival (OS), local relapse-free survival (LRFS), regional relapse-free survival (RRFS), distant metastasis failure-free survival (DMFS), and disease-free survival (DFS). The ranked data was analyzed by Wilcoxon rank sum test and sites of primary and recurrent tumor invasion were compared by McNemar test. Two-tailed $P$-values less than 0.05 were considered statistically significant.

\section{Results}

\section{Patient characteristics and survival}

Among the 382 patients, there were 279 males and 103 females. WHO types II and III were present in 83 and 287 patients, respectively. According to the 7th edition of AJCC Staging 
System, there were 94, 116, 68, and 104 cases of T1, T2, T3, and T4 disease, respectively. Within this sample, 154 patients $(40.3 \%)$ received induction chemotherapy, 141 patients $(36.9 \%)$ received concurrent chemotherapy, and 69 patients $(18.1 \%)$ received adjuvant chemotherapy. Patients' characteristics are listed in Table 1.

The median follow-up time was 61.1 months, with a range from 1-91 months. The 5-year OS, LRFS, RRFS, DMFS, and DFS were $86.9 \%, 96.7 \%, 99.1 \%, 93.3 \%$, and $82.6 \%$, respectively. A total of $12(3.1 \%)$ patients developed local recurrence in the primary area, three $(0.8 \%)$ patients experienced neck recurrence, and 23 (6.0\%) patients developed

Table I Patient characteristics

\begin{tabular}{|c|c|c|}
\hline & $\begin{array}{l}\text { Number } \\
\text { of patients }\end{array}$ & $\%$ \\
\hline \multicolumn{3}{|l|}{ Age, years } \\
\hline Median & 52 & \\
\hline Range & $14-93$ & \\
\hline \multicolumn{3}{|l|}{ Sex } \\
\hline Male & 279 & 73.0 \\
\hline Female & 103 & 27.0 \\
\hline \multicolumn{3}{|l|}{ Pathology } \\
\hline $\begin{array}{l}\text { Differentiated non-keratinizing } \\
\text { carcinoma (II) }\end{array}$ & 9 & 2.4 \\
\hline $\begin{array}{l}\text { Undifferentiated non- } \\
\text { keratinizing carcinoma (III) }\end{array}$ & 268 & 70.2 \\
\hline Non-keratinizing carcinoma (III) & 19 & 5.0 \\
\hline $\begin{array}{l}\text { Low differentiated squamous } \\
\text { cell carcinoma (II) }\end{array}$ & 74 & 19.4 \\
\hline Others & 12 & 3.1 \\
\hline \multicolumn{3}{|l|}{ T stage } \\
\hline TI & 94 & 24.6 \\
\hline $\mathrm{T} 2$ & 116 & 30.4 \\
\hline T3 & 68 & 17.8 \\
\hline T4 & 104 & 24.2 \\
\hline \multicolumn{3}{|l|}{ RLN } \\
\hline RLN (-) & 196 & 51.3 \\
\hline RLN (+) & 186 & 48.7 \\
\hline \multicolumn{3}{|l|}{ Radiotherapy } \\
\hline Median dose (Gy) & 68.2 & \\
\hline Range (Gy) & $52-77$ & \\
\hline \multicolumn{3}{|l|}{ Chemotherapy } \\
\hline No & 161 & 42.1 \\
\hline Induction & 24 & 6.3 \\
\hline Concurrent & 56 & 14.7 \\
\hline Induction+concurrent & 72 & 18.7 \\
\hline Concurrent+adjuvant & 11 & 2.9 \\
\hline Induction+adjuvant & 56 & 14.7 \\
\hline Induction+concurrent+adjuvant & 2 & 0.5 \\
\hline \multicolumn{3}{|l|}{ Boost } \\
\hline No & 349 & 91.4 \\
\hline Nasopharyngeal boost & 28 & 7.3 \\
\hline Nodal boost & 4 & 1.0 \\
\hline Nasopharyngeal and nodal boost & I & 0.3 \\
\hline
\end{tabular}

Abbreviation: RLN, retropharyngeal lymph nodes. distant metastasis. The most frequent sites of metastasis were the lung $(n=9,2.4 \%)$ and liver $(n=4,1.0 \%)$ (Table 2$)$.

\section{Dosimetric data}

Table 3 shows the DVH statistics for patients with local recurrence. An analysis of all the target volumes failed to identify any shortcomings, and only $0.5 \%$ of the gross tumor volume of primary tumor (GTV-P) and $0.8 \%$ of the clinical tumor volume of the high-risk region (CTV-P) received $<95 \%$ of the prescribed dose. The majority (96\%) of the GTV-P actually received more than $100 \%$ of the prescribed dose. A similar situation was found in CTV-P. The mean dose to the GTV-P was $70.4 \mathrm{~Gy}$ and to the CTV-P was $65.0 \mathrm{~Gy}$. Dose conformity with IMRT was excellent.

\section{Patterns of local recurrences}

In the 12 patients who developed local recurrences, there were two missing values because of the unavailability of the diagnostic image. As shown in Table 4, the median recurrence time was 15 months (10-59 months), and recurrence was mainly within 3 years after the first treatment (90\%), except for one case in 59 months. Among the 10 cases with local recurrence, seven $(70 \%)$ occurred within the $95 \%$ isodose lines and were considered in-field failures; two (20\%) were marginal, occurring in a steep dose gradient region at the unilateral margin of the high-dose planning target volume of high-risk region (PTV-C) volume, the other one $(10 \%)$ was outsidefield failure. The rate of marginal and out-of-field recurrence after a complete treatment response and in the whole cohort was $2 / 382(0.5 \%)$ and $2 / 382(0.3 \%)$, respectively.

\section{Time and location of onset of recurrence}

The time of onset of recurrence ranged from 10-59 months after radiotherapy, with an average of 28 months; eight cases $(80 \%)$ recurred within 3 years, and no patient had a recurrence more than 5 years after radiotherapy.

With the exception of anatomic sites on the midline, such as the base of the sphenoid bone and clivus, the bilateral NPC was defined by MRI as a tumor extending across the midline of the nasopharynx. Most patients had bilateral tumor

Table 2 Failure patterns of all patients

\begin{tabular}{lll}
\hline & Frequency & $\%$ \\
\hline Local recurrence & II & 30.6 \\
Distant metastases & $2 \mathrm{I}$ & 58.3 \\
Regional recurrence & 2 & 5.6 \\
Local recurrence and distant failures & $\mathrm{I}$ & 2.8 \\
Regional recurrence and distant failures & $\mathrm{I}$ & 2.8 \\
Total & 36 & 100.0 \\
\hline
\end{tabular}


Table 3 DVH statistics for patients of local recurrence

\begin{tabular}{lll}
\hline & GTV-P (range) & CTV-P (range) \\
\hline Volume (cc) & $71.60(30.46-135.52)$ & $466.10(306.17-609.30)$ \\
Dmin (cGy) & $6,143.87(5,542.00-6,471.00)$ & $3,308.38(1,289.80-5,468.30)$ \\
Dmax (cGy) & $7,350.32(6,956.00-7,956.00)$ & $7,352.38(6,956.10-7,956.60)$ \\
Dmean (cGy) & $7,042.25(6,733.00-7,589.00)$ & $6,500.09(6,343.00-6,791.30)$ \\
V95\% & $99.50(98.03-100)$ & $99.20(98.62-99.94)$ \\
V100\% & $96.00(88.00-99.00)$ & $96.00(94.55-97.68)$ \\
VII0\% & $0(0-0)$ & $28.00(0-53.27)$ \\
\hline
\end{tabular}

Abbreviations: DVH, dose-volume histogram; GTV-P, gross tumor volume of primary tumor; CTV-P, clinical tumor volume of the high-risk region; Dmax, maximum dose; Dmean, mean dose; Dmin, minimum dose; V95\%, percentage of volume receiving $>95 \%$ of the prescribed dose; VI00\%, percentage of volume receiving $>100 \%$ of the prescribed dose; $\mathrm{VII} \%$, percentage of volume receiving $>110 \%$ of the prescribed dose.

invasion into the mucous membrane of the nasopharynx $(90 \%$ at initial diagnosis and $70 \%$ at recurrence). Nevertheless, as shown in Table S1, most anatomic sites were at low risk of concurrent bilateral tumor invasion at initial diagnosis and at recurrence.

\section{Discussion}

Owing to widespread application of IMRT and the use of combined chemotherapy, the local control of NPC patients is excellent, which has been proven by treatment outcomes from various centers. ${ }^{8,12-14}$ In our study, the 5-year LRFS for patients with stage T1-4 was $98.8 \%, 97.6 \%, 96.2 \%$, and $90.0 \%$, respectively $(P=0.032)$, which indicated that a higher $\mathrm{T}$ category was associated with poorer LRFS. Whereas, as shown in Table 5, 40\% of the recurrence occurred in patients who were diagnosed with $\mathrm{T} 1$ or $\mathrm{T} 2$ at the initial treatment. Multiple studies have indicated that hypoxia plays an important role in affecting the tumor microenvironment, and is the ultimate cause of radioresistance, which results in worse local control. ${ }^{15-17}$ In addition, weight loss and the contraction of primary tumor during the course of RT can result in changes in body contour and target volume, which will affect the dosage distribution and lead to in-field failure. ${ }^{18}$ Qi et al ${ }^{19}$ investigated the relationships between critical weight loss and long-term survival by categorizing weight change into critical weight loss (CWL) and non-critical weight loss (Non-CWL) in 2,399 NPC patients. They found that, in the IMRT cohort, CWL was significantly associated with a lower OS and failure-free survival rates ( $P=0.04$ and 0.04 , respectively). The mean relative weight loss during treatment was $13.2 \%$ $(+6.0 \%)$, and a significant correlation between the volume reduction and weight loss was observed $(P=0.01)$.

In our study, there were two "marginal failures" occurring in a steep dose gradient region at the unilateral margin of the high-dose PTV-C volume (patient 3 and patient 6). CTV delineation of the primary lesion plays an important role in local tumor control and normal tissue protection, and the current definitions are largely derived from our experience of $3 \mathrm{D}$ or conventional radiation fields, but with a different dose gradient and a lack of individualization. Although RTOG 0225 and $0615^{11,20}$ have provided a practical reference for the delineation of CTV-1 for NPC, the optimal definition of CTV for the primary disease has not been determined, and the individual CTV should be delineated both by distance from GTV and the

Table 4 Details of local recurrence patients

\begin{tabular}{|c|c|c|c|c|c|c|c|c|c|c|c|}
\hline \multirow[t]{2}{*}{ Patient } & \multirow[t]{2}{*}{ Gender } & \multirow{2}{*}{$\begin{array}{l}\text { Recurrence } \\
\text { period }(\mathrm{m})\end{array}$} & \multirow{2}{*}{$\begin{array}{l}\text { Initial } \\
\text { T stage }\end{array}$} & \multirow{2}{*}{$\begin{array}{l}\text { Initial- } \\
\text { GTV (cc) }\end{array}$} & \multirow{2}{*}{$\begin{array}{l}\text { Location of the } \\
\text { recurrence volume }\end{array}$} & \multicolumn{5}{|c|}{ DVH statistics to recurrence volume } & \multirow[t]{2}{*}{ Type } \\
\hline & & & & & & $\begin{array}{l}\text { GTVr } \\
\text { (cc) }\end{array}$ & $\begin{array}{l}\text { Dmin } \\
(c G y)\end{array}$ & $\begin{array}{l}\text { Dmax } \\
\text { (cGy) }\end{array}$ & $\begin{array}{l}\text { Dmean } \\
\text { (cGy) }\end{array}$ & V95\% & \\
\hline I & M & 14.8 & $\mathrm{~T} 2$ & 39.00 & GTV & 8.80 & $6,588.0$ & $7,111.00$ & $6,855.0$ & 100 & In-field \\
\hline 2 & M & 16.4 & $\mathrm{~T} 2$ & 30.46 & GTV & 23.45 & $6,171.0$ & $7,042.00$ & $6,820.0$ & 99.7 & In-field \\
\hline 3 & $\mathrm{~F}$ & 33.1 & TI & 46.15 & Marginal to CTV & 9.35 & $5,775.1$ & $7,182.60$ & $6,782.6$ & 94.6 & Marginal \\
\hline 4 & M & 59 & $\mathrm{~T} 2$ & 80.67 & GTV & 19.06 & $6,489.9$ & $6,956.10$ & $6,767.4$ & 100 & In-field \\
\hline 5 & $\mathrm{~F}$ & 6 & $\mathrm{~T} 4$ & 82.43 & GTV & 9.80 & $7,015.9$ & $7,544.10$ & $7,307.0$ & 100 & In-field \\
\hline 6 & $M$ & 28 & T3 & 88.50 & Marginal to CTV & 43.55 & $5,7 \mid 0.0$ & $7,637.02$ & $7,222.3$ & 94.5 & Marginal \\
\hline 7 & $M$ & 10 & $\mathrm{~T} 4$ & 99.32 & GTV & 11.49 & $7,283.7$ & $7,953.90$ & $7,683.5$ & 100 & In-field \\
\hline 8 & $M$ & 35 & $\mathrm{~T} 4$ & 91.35 & GTV & 27.99 & $6,506.7$ & $7,718.80$ & $7,402.2$ & 99.9 & In-field \\
\hline 9 & $M$ & 12 & $\mathrm{~T} 4$ & 135.52 & GTV & 23.06 & $6,701.7$ & $7,575.10$ & $7,213.9$ & 100 & In-field \\
\hline 10 & $M$ & 12 & $\mathrm{~T} 2$ & 49.00 & Outside CTV & 18.03 & 328.3 & $6,668.90$ & $2,598.8$ & 3.2 & Out-field \\
\hline
\end{tabular}

Notes: In-field refers to $95 \%$ of the recurrence volume receiving more than $95 \%$ of the prescribed dose. Marginal refers to $20 \%-95 \%$ of the recurrence volume receiving $95 \%$ of the prescribed dose. Outside refers to less than $20 \%$ of the recurrence volume receiving $95 \%$ of the prescribed dose.

Abbreviations: CTV, clinical target volume; DVH, dose-volume histogram; F, female; GTV, gross tumor volume; GTVr, the recurrent tumor volume; M, male; Dmin, minimum dose; Dmax, maximum dose; Dmean, mean dose; V95\%, percentage of volume of failure to receive at least $95 \%$ of prescribed total dose. 
Table 5 Comparison between the delineation of CTV in our center, in the Cancer Hospital of Fujian Medical University, and those of the RTOG

\begin{tabular}{|c|c|c|c|c|}
\hline Region & RTOG 0225 & RTOG 0615 & $\begin{array}{l}\text { Cancer Hospital of Fujian Medical } \\
\text { University }\end{array}$ & $\begin{array}{l}\text { Current protocol } \\
\text { in our center }\end{array}$ \\
\hline Sphenoid sinus & Inferior part & $\begin{array}{l}\text { Inferior part (in T3-T4 disease, } \\
\text { the entire sphenoid sinus) }\end{array}$ & $\begin{array}{l}\text { Inferior part (in sphenoid sinus involved } \\
\text { disease, the entire sphenoid sinus) }\end{array}$ & Inferior part \\
\hline Ethmoid sinus & Not included & Not included & Posterior & Posterior I/2 \\
\hline Nasal cavity & Posterior $1 / 3$ & Posterior $1 / 4$ to $1 / 3$ & 5-mm anterior to posterior nasal aperture & Posterior $1 / 3$ \\
\hline Maxillary sinus & Posterior I/3 & Posterior $1 / 4$ to $1 / 3$ & 5-mm anterior to maxillary mucosa & Posterior I/3 \\
\hline Clivus & Entire & Anterior $1 / 2$ to $2 / 3$ & Anterior $1 / 3$ & Entire \\
\hline $\begin{array}{l}\text { Retropharyngeal } \\
\text { lymph nodes }\end{array}$ & $\begin{array}{l}\text { Base of skull to cranial } \\
\text { edge of the hyoid }\end{array}$ & $\begin{array}{l}\text { Base of skull to cranial edge of } \\
\text { the hyoid }\end{array}$ & $\begin{array}{l}\text { Base of skull to cranial edge of the second } \\
\text { cervical vertebra }\end{array}$ & $\begin{array}{l}\text { Base of skull to cranial } \\
\text { edge of the hyoid }\end{array}$ \\
\hline
\end{tabular}

Abbreviations: CTV, clinical target volume; RTOG, Radiation Therapy Oncology Group.

patterns of local extension. In 2009, Liang ${ }^{21}$ analysed the data of 943 NPC patients who underwent MRI of the nasopharynx and neck. With reviewing by two radiologists, they found that most anatomic sites surrounding the nasopharynx were at low risk of concurrent bilateral tumor invasion $(<10 \%)$, local disease spreads stepwise from proximal sites to more distal sites, and that a skip pattern of local extension was unusual. Therefore, they believed that, when the tumor invades on one side of the nasopharynx, the bilateral anatomic sites at high risk should be included in the CTV, whereas the sites at medium or low risk and contralateral to the tumor invasion area should be excluded from the CTV. In 2014, Lin $^{22}$ analyzed the data of 414 NPC patients who were treated with IMRT to define CTV through GTV plus 5-10-mm margin in different directions and encompassed the entire nasopharyngeal mucosa plus 5-mm submucosal volume. With 60 months of follow-up, the 5-year OS, DFS, and local control were $80 \%, 77 \%$, and $95 \%$, respectively. No increase of local recurrence was associated with the limited margins used in the strategy. For stage T4 disease, margins in all six directions were significantly smaller than that of the whole group of patients. Therefore, they concluded that CTV-1, which included GTV plus a 5-10-mm margin and encompassed the entire nasopharyngeal mucosa plus 5-mm submucosal volume might be feasible (Table 5). This finding suggested that the target volumes used in the Cancer Hospital of Fujian Medical University were adequate. On the other hand, our institution adopted a symmetric coverage of CTV delineation and attained excellent local control as well. By analyzing the location of relapse site, we presented our proposed guidelines on CTV target volume delineation. As it was shown in our study that most anatomic sites were at low risk of concurrent bilateral tumor invasion, and most local relapse sites located just the same site of primary tumor, we suggest that contralateral to the tumor invasion area should be excluded from the CTV.
As shown in Table 4, most of the local relapses (90\%) in our study occurred within the high dose region, and only one out-field local failure was observed in this study. Patient 10 had T2N0 disease with parapharyngeal space invasion, and he was treated with radiation alone. However, he complained of a foreign body in the left nasal cavity 1 year after primary treatment. The fine-needle aspiration confirmed the presence of local recurrence and the pathology was (left nasal cavity) undifferentiated non-keratinizing carcinoma (Figure 1). A retrospective review of the pretreatment MRI did not show any disease at the paranasal sinus. He underwent salvage chemotherapy (four cycles of GP) and IMRT ( $66 \mathrm{~Gy} / 33 \mathrm{Fx}$ ) and he is still surviving without evidence of disease for 60.2 months. A similar case has been reported; $\mathrm{Ng}$ et $\mathrm{al}^{4}$ reported a patient had both local and regional failures 1 year after primary treatment, and the sites of local recurrence were predominantly at the maxillary and ethmoid sinus. At the primary treatment, he had T3N2 disease with sphenoid sinus invasion, and pretreatment MRI did not show any disease at any of these paranasal sinuses. The ethmoid sinus is a highly unusual site of recurrence of NPC, and there remains a question over whether this could represent a new primary tumor. ${ }^{21}$ Both of these recurrences occurred within 1 year of primary treatment, which suggests that they do not represent new primary tumors. Longer follow-up and a large sample of study are needed to identify whether the posterior ethmoid sinus should be included in the high-risk CTV.

There were several limitations of this study. First, the retrospective nature of this analysis certainly constitutes a pitfall of this study, although a relatively large number of contiguous and nonselected patients were included. Second, image fusion and dose-analysis cannot guarantee $100 \%$ accuracy because of the influence of various factors such as position, time, and surgery. 
A
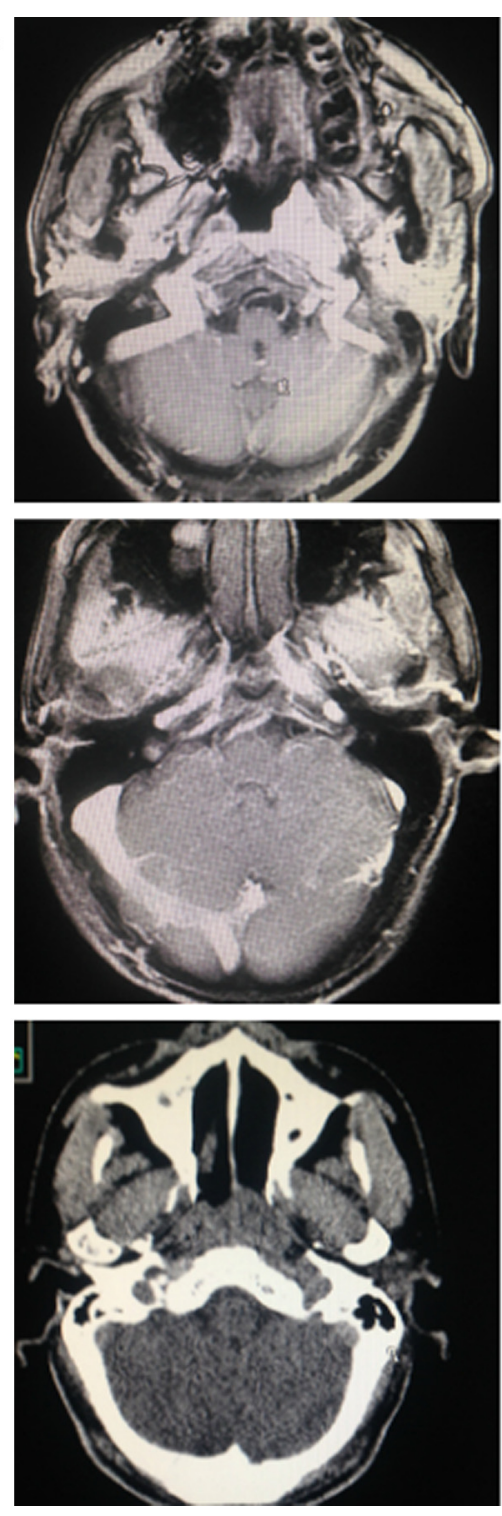

B
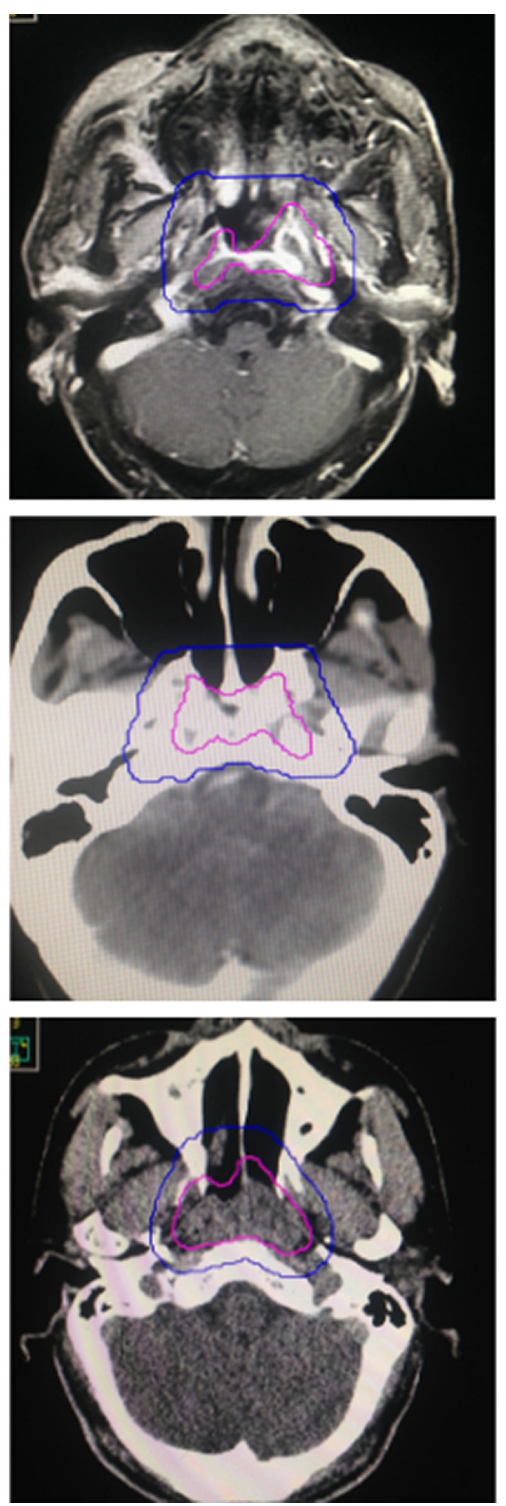
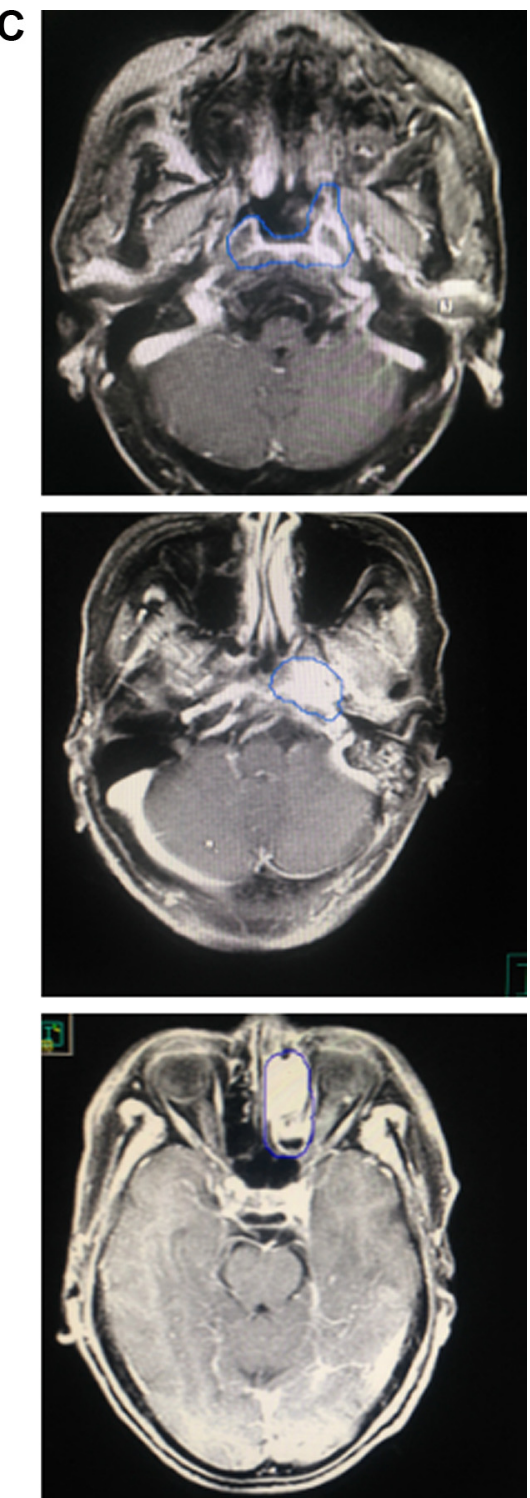

Figure I Disease extent for patients having local failures.

Notes: (A) In-field failure. (B) Marginal failure. (C) Outside-field failure. Left, pretreatment MRI/CT. Middle, the recurrent tumor volumes were transferred from the diagnostic MRI/CT at the time of recurrence to the planning CT to show doses delivered to the recurrence sites. Right, MRI/CT at time of failure. Blue line indicates clinical tumor volume of the high-risk region; purple line indicates gross tumor volume of primary tumor.

Abbreviations: MRI, magnetic resonance imaging; CT, computed tomography.

\section{Conclusion}

Our study investigated the local failure patterns of NPC patients after IMRT in a relatively large number of patients and found that IMRT with elective neck irradiation provides excellent local control for NPC patients without cervical lymph node metastasis. In-field failures are the main patterns for local recurrence, and the radioresistant subvolumes within the GTV need to be identified. Most local relapse sites located just the same site of primary tumor and most anatomic sites were at low risk of concurrent bilateral tumor invasion, so we proposed suggestions for reduction of target volume during IMRT treatment for NPC patients.

\section{Acknowledgments}

We acknowledge the support of the Department of Radiation Oncology of Fudan University Shanghai Cancer Center. The views expressed in this publication are those of the authors.

\section{Author contributions}

Chunying Shen, Tingting Xu, Weiwei Li, and Chaosu Hu participated in the treatment and planning, Yujiao Li and Xiaomin Ou contributed to the data collection, performed the statistical analysis, and drafted the manuscript. All authors contributed toward data analysis, drafting and revising the paper and agree to be accountable for all aspects of the work. 


\section{Disclosure}

The authors report no conflicts of interest in this work.

\section{References}

1. Wang J, Shi M, Hsia Y. Failure patterns and survival in patients with nasopharyngeal carcinoma treated with intensity modulated radiation in Northwest China: a pilot study. Radiat Oncol. 2012;7:2.

2. Ou X, Zhou X, Shi Q, et al. Treatment outcomes and late toxicities of 869 patients with nasopharyngeal carcinoma treated with definitive intensity modulated radiation therapy: new insight into the value of total dose of cisplatin and radiation boost. Oncotarget. 2015;6: 38381-38397.

3. Zhao W, Lei H, Zhu X, Li L, Qu S, Liang X. Investigation of long-term survival outcomes and failure patterns of patients with nasopharyngeal carcinoma receiving intensity-modulated radiotherapy: a retrospective analysis. Oncotarget. 2016;7:86914-86925.

4. Ng WT, Lee MC, Hung WM, et al. Clinical outcomes and patterns of failure after intensity-modulated radiotherapy for nasopharyngeal carcinoma. Int J Radiat Oncol Biol Phys. 2011;79:420-428.

5. Dawson LA, Anzai Y, Marsh L, et al. Patterns of local-regional recurrence following parotid-sparing conformal and segmental intensitymodulated radiotherapy for head and neck cancer. Int $J$ Radiat Oncol Biol Phys. 2000;46:1117-1126.

6. Li JX, Huang SM, Jiang XH, et al. Local failure patterns for patients with nasopharyngeal carcinoma after intensity-modulated radiotherapy. Radiat Oncol. 2014;9:87.

7. Kong F, Ying H, Du C, et al. Patterns of local-regional failure after primary intensity modulated radiotherapy for nasopharyngeal carcinoma. Radiat Oncol. 2014;9:60.

8. Mao YP, Tang LL, Chen L, et al. Prognostic factors and failure patterns in non-metastatic nasopharyngeal carcinoma after intensity-modulated radiotherapy. Chin J Cancer. 2016;35:103.

9. Lin S, Pan J, Han L, Zhang X, Liao X, Lu JJ. Nasopharyngeal carcinoma treated with reduced-volume intensity-modulated radiation therapy: report on the 3-year outcome of a prospective series. Int J Radiat Oncol Biol Phys. 2009;75:1071-1078.

10. Orlandi E, Tomatis S, Potepan P, et al. Critical analysis of locoregional failures following intensity-modulated radiotherapy for nasopharyngeal carcinoma. Future Oncol. 2013;9:103-114.
11. RTOG. Clinical Trials. Study Number 0225. 2017. Available from: https://www.rtog.org/ClinicalTrials/ProtocolTable/StudyDetails. aspx? study $=0225$. Accessed November 29, 2017.

12. Ou X, Shen C, Kong L, et al. Treatment outcome of nasopharyngeal carcinoma with retropharyngeal lymph nodes metastasis only and the feasibility of elective neck irradiation. Oral Oncol. 2012;48:1045-1050.

13. Setton J, Han J, Kannarunimit D, et al. Long-term patterns of relapse and survival following definitive intensity-modulated radiotherapy for nonendemic nasopharyngeal carcinoma. Oral Oncol. 2016;53:67-73.

14. Zeng L, Sun XM, Chen CY, et al. Comparative study on prophylactic irradiation to the whole neck and to the upper neck for patients with neck lymph node-negative nasopharyngeal carcinoma. Head Neck. 2014;36:687-693.

15. Kumar P. Impact of anemia in patients with head and neck cancer. Oncologist. 2000;5(Suppl 2):13-18.

16. Brizel DM, Dodge RK, Clough RW, Dewhirst MW. Oxygenation of head and neck cancer: changes during radiotherapy and impact on treatment outcome. Radiother Oncol. 1999;53:113-117.

17. Karar J, Maity A. Modulating the tumor microenvironment to increase radiation responsiveness. Cancer Biol Ther. 2009;8:1994-2001.

18. Li G, Gao J, Liu ZG, et al. Influence of pretreatment ideal body weight percentile and albumin on prognosis of nasopharyngeal carcinoma: long-term outcomes of 512 patients from a single institution. Head Neck. 2014;36:660-666.

19. Zeng Q, Shen LJ, Guo X, Guo XM, Qian CN, Wu PH. Critical weight loss predicts poor prognosis in nasopharyngeal carcinoma. BMC Cancer. 2016;16:169.

20. RTOG. Clinical Trials. Study Number 0615. 2017.

21. Liang SB, Sun Y, Liu LZ, et al. Extension of local disease in nasopharyngeal carcinoma detected by magnetic resonance imaging: improvement of clinical target volume delineation. Int J Radiat Oncol Biol Phys. 2009; 75:742-750.

22. Lin S, Pan J, Han L, et al. Update report of nasopharyngeal carcinoma treated with reduced-volume intensity-modulated radiation therapy and hypothesis of the optimal margin. Radiother Oncol. 2014;110: 385-389. 


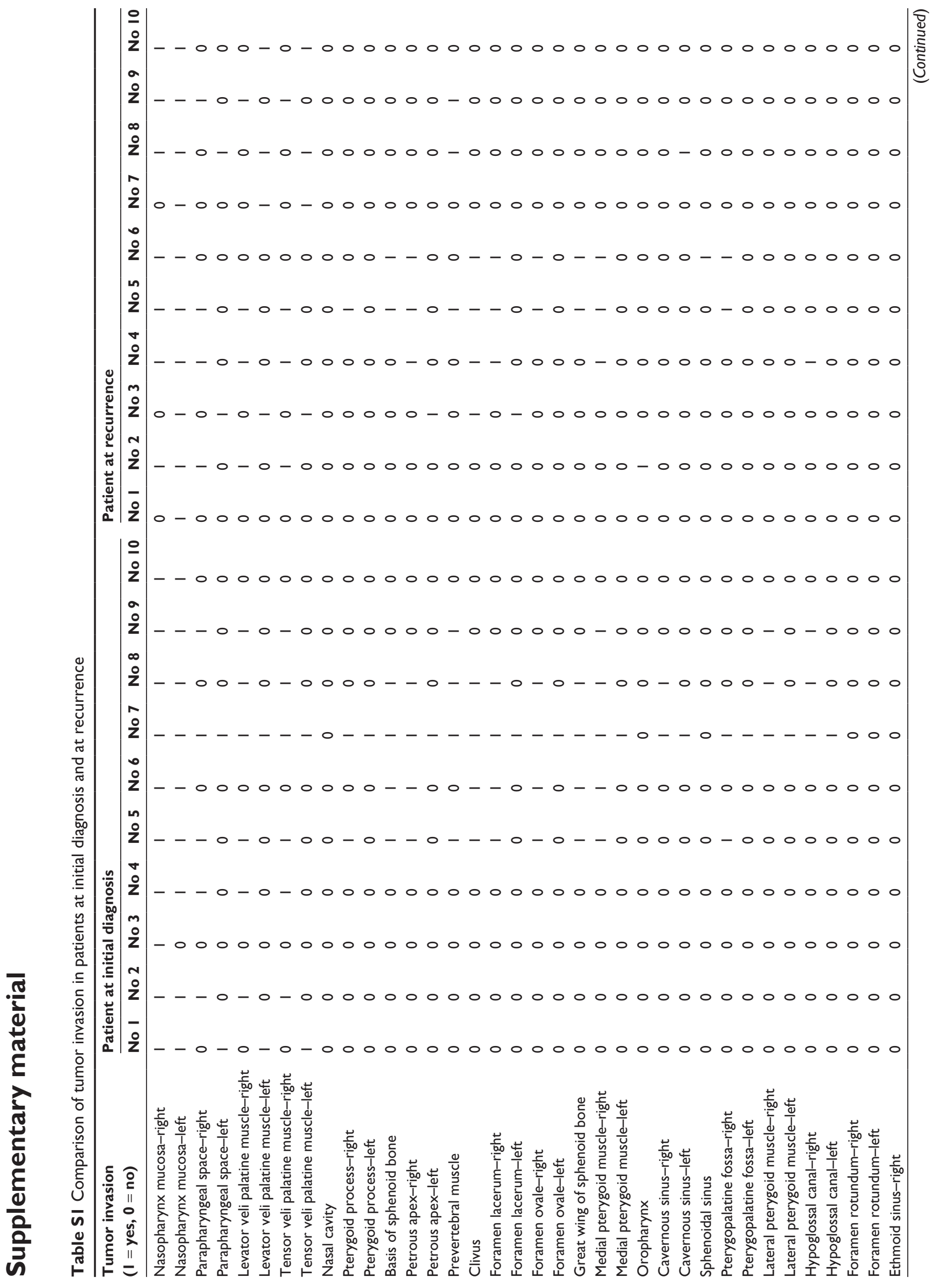




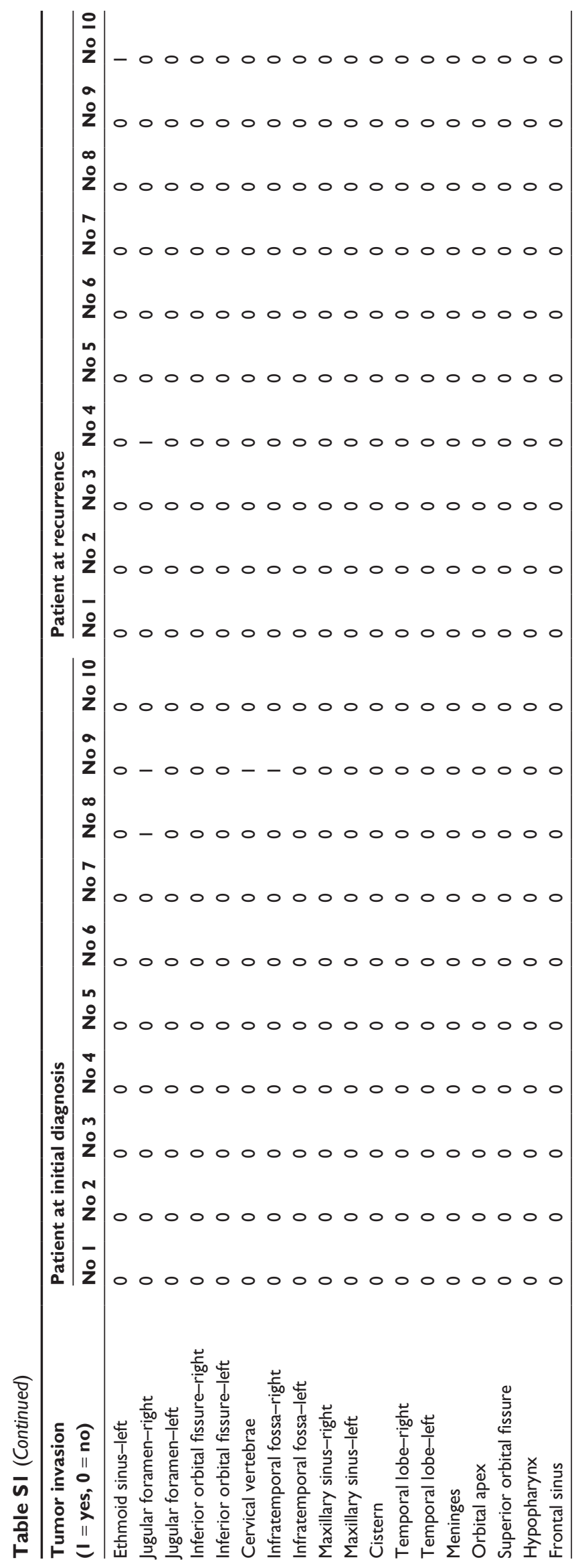


OncoTargets and Therapy

\section{Publish your work in this journal}

OncoTargets and Therapy is an international, peer-reviewed, open access journal focusing on the pathological basis of all cancers, potential targets for therapy and treatment protocols employed to improve the management of cancer patients. The journal also focuses on the impact of management programs and new therapeutic agents and protocols on

patient perspectives such as quality of life, adherence and satisfaction. The manuscript management system is completely online and includes a very quick and fair peer-review system, which is all easy to use. Visit http://www.dovepress.com/testimonials.php to read real quotes from published authors.

Submit your manuscript here: http://www.dovepress.com/oncotargets-and-therapy-journal 